\title{
Malnutrition and the Risk of Catastrophic Health Expenditure in Nigeria
}

\author{
Ryoko Sato ${ }^{1}$ \\ ${ }^{1}$ Harvard T.H. Chan School of Public Health, Boston, USA \\ Correspondence: Center for Health Decision Science, Harvard T.H. Chan School of Public Health, 90 Smith St, \\ Boston 02120, USA. Tel: 1-202-290-5064. E-mail: rsato@hsph.harvard.edu; ryokos1226@gmail.com
}

Received: October 18, 2021 Accepted: November 26, 2021 Online Published: December 6, 2021

doi:10.5539/gjhs.v14n1p36 URL: https://doi.org/10.5539/gjhs.v14n1p36

\begin{abstract}
Background: Malnutrition among children not only affects their health consequences but also does it burden their households' finance especially in developing countries. This study evaluates the household risk of catastrophic health expenditure (CHE) due to malnutrition treatments among malnourished children in Nigeria, according to zones and wealth quintiles. We simulate the CHE risk among households with malnourished children who seek treatment.

Methods: The CHE risk due to malnutrition among treated was computed based on 1) the out-of-pocket (OOP) expenditure and indirect costs associated with malnutrition treatment, and 2) household consumption expenditures. I derived the CHE risk associated with malnutrition across zones and wealth quintiles in Nigeria, using secondary data sources for healthcare utilization, OOP expenditures, and consumption expenditures.

Results: There was a large variation of CHE risk according to zones and wealth quintiles. Among the poorest households, those in northeast and northwest would have the highest risk of CHE, up to 59 and $47 \%$, while those in southwest would have the lowest risk of $14 \%$. For all zones, as the wealth increases, the CHE risk would decrease. There would be zero or very little CHE risk among the richest households in any zones.
\end{abstract}

Interpretation: Nutrition interventions will help malnourished children improve their health status. However, we should also be wary about the financial consequences of the treatment that households should bear.

Keywords: catastrophic health expenditure, malnutrition, Nigeria

\section{Introduction}

Malnutrition is the leading cause of deaths in developing countries: up to $45 \%$ of deaths among children under 5 years old are attributed to factors related to nutrition (WHO, 2020). Malnutrition also affects people in the long term (for example, see Mwene-Batu et al., 2020). In Nigeria, it is estimated that about 2 million children have severe acute malnutrition but only $20 \%$ of them receive treatment (UNICEF, 2021).

Furthermore, malnutrition can have a substantial negative impact not only on population's health, but also on people's finance due to the financial burden of malnutrition treatment and diminished productivity (Njuguna et al., 2020). Thus, treatment of malnutrition at earlier stage is crucial to minimize the long-term health and financial burdens among children and their households.

This paper evaluates the financial consequence of malnutrition treatment in Nigeria. In particular, I focus on the incidence of catastrophic health expenditures (CHE) as an indicator of financial burden as a consequence of malnutrition treatment in this paper. CHE is defined as out-of-pocket (OOP) medical expenditures surpassing a certain threshold of income or consumption expenditures. I investigate differences in CHE risks according to zones and wealth quintiles.

\section{Methodology}

In this section, I present the main approach to estimate the risk of facing CHE as a consequence of children's malnutrition treatment, among malnourished children, in Nigeria. In this study, I assumed that malnutrition was treated through Community-based Management of Acute Malnutrition (CMAM) (Frankel et al., 2015). Thus, the cost estimates for the malnutrition treatment were based on CMAM intervention. Although many malnourished children have no access to treatment for malnourishment in reality, this paper examined the financial consequence 
of malnutrition treatment, assuming children had access to one.

\subsection{Modeling Risk of CHE Due to Malnutrition}

Generally, for a household, the risk of facing CHE, conditional on being malnourished, would depend on: (i) the likelihood of healthcare utilization to treat malnutrition; (ii) the amount of OOP health expenditures and indirect costs associated with malnutrition treatment; and (iii) the level of household total consumption expenditures. This study focused on the best scenario where all the households with malnourished children received the treatment. Thus, CHE risk among treated was independent on the factor (i).

Illness-related costs associated with treatment of malnutrition included several components: OOP direct medical costs, OOP transportation costs to health facilities, and indirect costs (Table 1). I closely followed the study from Frankel et al. (2015) to refer to the costs related to the malnutrition treatment. According to their study based on Community-based Management of Acute Malnutrition (CMAM), it cost $\$ 40.0$ for inpatient treatment while it cost $\$ 6.3$ for outpatient treatment. Transportation costs for both inpatient and outpatient visit were assumed to be $\$ 6.3$. Indirect costs had two components: an estimated "wage rate" and the number of workdays lost due to inpatient stays. For the wage rate, I used mean annual consumption expenditures per adult equivalent according to zone and wealth quintile. The average bed day for inpatient treatment was assumed to be 8 days from malnutrition treatment study (Daures et al., 2020). We also referred to Daures et al. (2020) for the ratio of inpatient and outpatient visits: $13.7 \%$ for inpatient visits and the remaining $86.3 \%$ were outpatient visits.

Table 1. Cost information

\begin{tabular}{lc}
\hline & USD \$ \\
\hline Inpatient costs (total) & 39.956 \\
Outpatient costs (total) & 6.343 \\
Transportation costs to clinic & 6.343 \\
\hline Average bed days (days) & 8 \\
$\%$ hospitalization & 13.7 \\
$\%$ outpatient & 86.3 \\
\hline
\end{tabular}

Sources of data: inpatient costs, inpatient costs, transportation costs to clinic (Frankel et al., 2015); average bed days, \% hospitalization, \% outpatient (Daures et al., 2020)

The annual consumption expenditure was drawn from Nigeria Living Standards Survey (NLSS), which was the national representative survey conducted in 2018 to 2019 (Nigeria National Bureau of Statistics, 2019). I calculated the average annual consumption per capita according to zone and wealth quintile (Figure 1). 


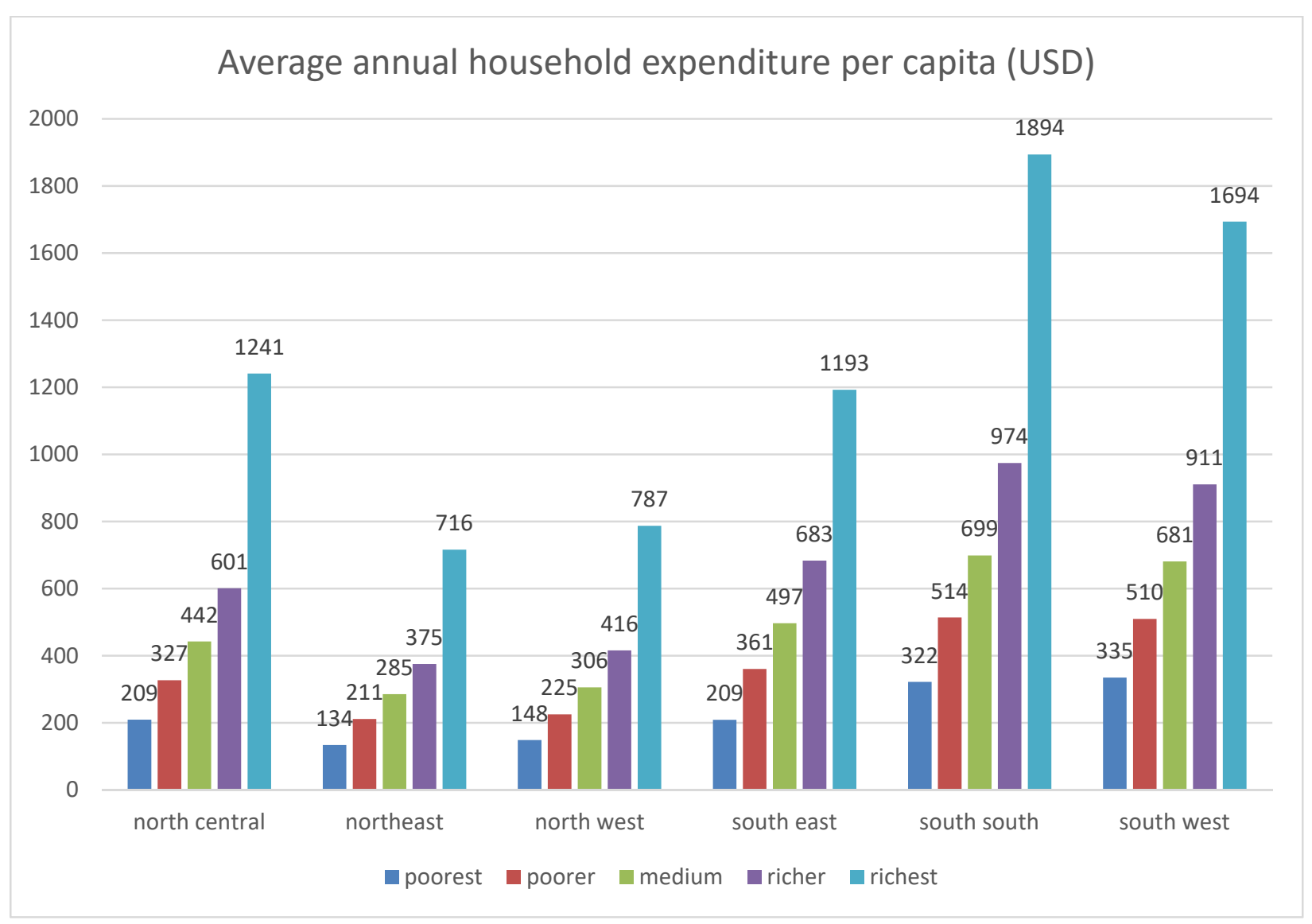

Figure 1. Distribution of household expenditure per capita in each zone across wealth quintiles

With the information above, I could derive a risk to facing a CHE case as a result of malnutrition treatment among households with malnourished children. Employing the methods from Riumallo-Herl et al. (2018), I estimated the number of cases of CHE associated with malnutrition treatment in each zone at each wealth quintile in Nigeria. A case of CHE for malnutrition was counted when total patient-incurred expenditures, which is the total OOP costs of medical, transportation, and indirect costs, surpassed $10 \%$ of total annual consumption expenditures.

I reported, per wealth quintile in each zone of Nigeria, on the risk of facing CHE in treating malnutrition. Wealth quintiles were poorest, poorer, medium, richer, and richest. There are six zones in Nigeria: north central, northeast, northwest, southeast, south south, and southwest.

As indicators of malnutrition using the existing data for nationally representative data, the prevalence of severe wasting and severe stunting were evaluated across wealth quintiles in each zone. Although many nutritional interventions, including CMAM, used the indicator of wasting and Mid-Upper Arm Circumference (MUAC), I present the prevalence of wasting and stunting due to the data unavailability of MUAC measurement in Nigeria dataset.

All simulations were conducted using R statistical software (version 3.6.0) and STATA (version 15.1).

\section{Results}

The estimation of CHE risks depended on the amount of OOP expenditure and time losses for disease treatment, and the consumption expenditures of households. Figure 1 presents the distribution of consumption expenditure according to zone and wealth quintile. There were variations in the distribution of consumption expenditure according to zones.

Northeast zone had the lowest consumption expenditure for all the wealth quintiles. The poorest households in the northeast on average spent $\$ 134$ annually for consumption, while the richest households spent $\$ 716$. Northwest also had the low level of consumption expenditure. On the other hand, south south and south west zones were the richest zones in Nigeria: the poorest households in these zones spent about $\$ 330$ for consumption and this was much higher than the expenditure level among the medium households in north east, $\$ 285$. The richest households 
in south south zone spent the most, $\$ 1,894$.

Figure 2 presents the distribution of children with malnutrition using severe wasting and severe stunting measurement, in each zone and wealth quintile. Across Nigeria, the prevalence of severe wasting did not exceed $4 \%$ in all wealth quintile in all zones. The poorest in northeast and northwest had the highest proportion of children with severe wasting: $3.58 \%$ and $3.6 \%$ respectively. The clear pattern of the gradients of the prevalence in the severe wasting from the poorest to the richest was not observed. On the other hand, the prevalence of severe stunting was high, especially in northeast and northwest; $20.5 \%$ and $26.6 \%$ respectively. For the severe stunting, its prevalence became lower as the wealth level got higher for almost all the zones.

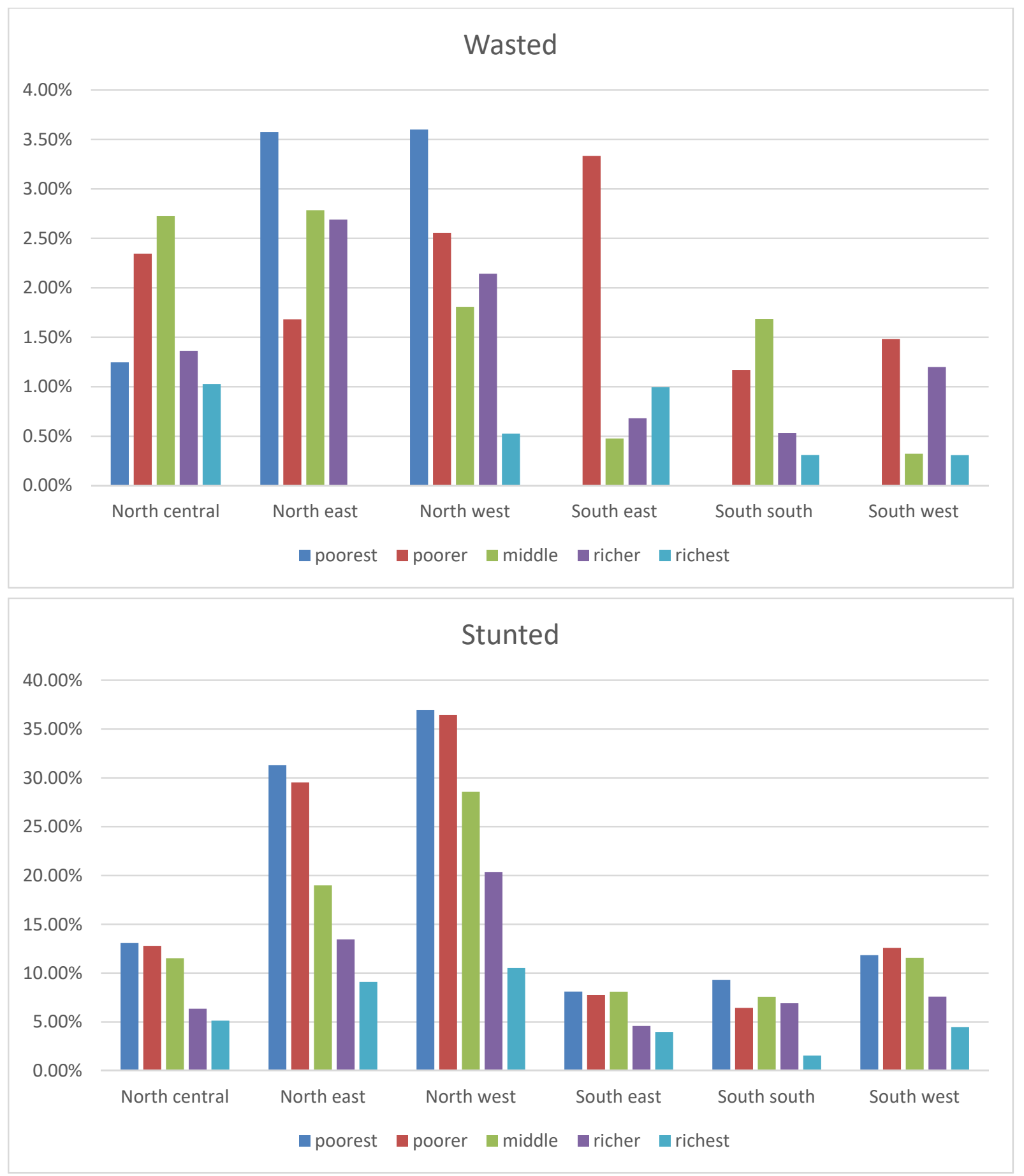

Figure 2. Prevalence of malnutrition in each zone across wealth quintiles

Notes. Wasted: weight for height z-score $<-3$; Stunted: height for age $<-3$ 
Figure 3 and Table 2 present the variations of the CHE risk due to malnutrition treatment across zone and wealth quintiles. On average, households in the northeast would have the highest CHE risk of $21.8 \%$, while households in south south and southwest would have the lowest CHE risk of 6.9 to $7.0 \%$.

Among the poorest households, those in northeast and northwest would have the highest risk, $58.6 \%$ and $47.0 \%$ respectively, while those in south south and southwest would have the lowest risk; $14.9 \%$ and $14.4 \%$. The CHE risk was almost non-existent ( 0 or less than $1 \%$ ) among the richest in all zones except northeast and northwest. Richest households in northeast and northwest still had 7 to $9 \%$ of CHE risk.

For all zones, as the wealth increases, the CHE risk would decrease. However, the reduction gradient from the poorest to poorer and to medium differed by zone. The CHE risk decreased drastically from the poorest to the poorer wealth category in northeast and northwest zones. For example, the CHE risk was $58.6 \%$ in northeast among the poorest but it decreased to $14.5 \%$ among the poorer. This CHE risk among the poorer in northeast was not much different from CHE risks among the poorer in other zones. We observed this catch-up among the poorer in northwest zone as well.

Table 2. CHE risk of malnutrition treatment in each zone across wealth quintiles

\begin{tabular}{lrrrrrr}
\hline & North central & North east & North west & South east & South south & South west \\
\hline poorest & $22.5 \%$ & $58.6 \%$ & $47.0 \%$ & $24.6 \%$ & $14.9 \%$ & $14.4 \%$ \\
poorer & $13.7 \%$ & $14.5 \%$ & $13.8 \%$ & $13.6 \%$ & $12.5 \%$ & $12.6 \%$ \\
middle & $13.4 \%$ & $13.7 \%$ & $13.7 \%$ & $12.8 \%$ & $6.5 \%$ & $7.2 \%$ \\
richer & $10.2 \%$ & $13.6 \%$ & $13.5 \%$ & $7.1 \%$ & $0.5 \%$ & $0.9 \%$ \\
richest & $0.9 \%$ & $8.6 \%$ & $7.0 \%$ & $0.3 \%$ & $0.0 \%$ & $0.0 \%$ \\
\hline average & $12.1 \%$ & $21.8 \%$ & $19.0 \%$ & $11.7 \%$ & $6.9 \%$ & $7.0 \%$ \\
\hline
\end{tabular}

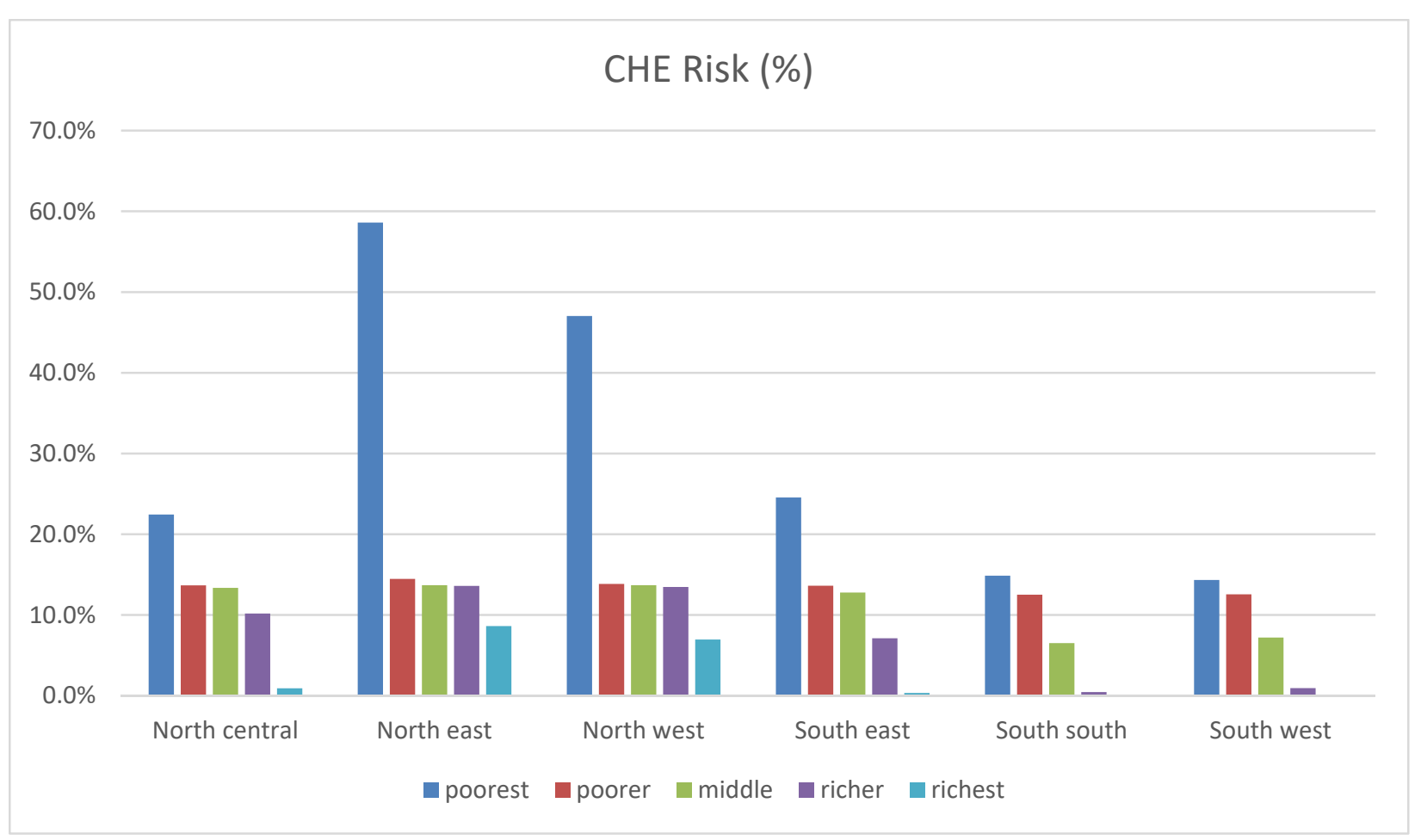

Figure 3. CHE risk of malnutrition treatment in each zone across wealth quintiles

\section{Discussion}

This paper evaluated the risk of CHE for malnutrition treatment according to the wealth quintile in each zone in Nigeria. The analysis was based on secondary data sources on OOP expenditure, time and wage losses for disease 
treatment, as well as on the consumption expenditure.

This modelling study found that the risk to CHE due to malnutrition treatment would vary greatly according to wealth quintiles and zones. The variation of CHE risk across six zones mostly coincided with the variation of consumption expenditure across these zones: if the average consumption expenditure in a zone was higher, then the CHE risk in the zone was lower. The average consumption expenditure was the lowest in northeast and northwest zones, and the CHE risk in these 2 zones was the highest: about $20 \%$. On the other hand, the average consumption was the highest in south south and south west, and the CHE risk in these zones was the lowest: about $7 \%$. This finding was based on that richer households had less chance of experiencing the catastrophic expenditure from malnutrition treatment as compared to poorer households who had tighter budget constraints. Overall, the CHE risk among the richest was almost non-existent across all zones except northeast and northwest. This finding was also consistent with the distribution of the total consumption among the richest across zones.

The gradient of the reduction of CHE risk from the poorest to the poorer households differed by zones. Northeast and northwest zones observed the great CHE risk among the poorest but the risk reduced greatly for the poorer to the level that was similar to CHE risks for other zones.

The findings of the paper are consistent with the literature: it has been found that the majority of OOP expenditure is for outpatient visits and medicines including the ones for malnutrition treatment (Yerramilli et al., 2018). However, the study to evaluate the CHE due to malnutrition is extremely scarce. This paper is the first to evaluate the extent of CHE due to malnutrition.

This study has some limitations. There was no data available to measure the prevalence of malnutrition using MUAC, which was increasingly common, using nationally representative samples in Nigeria. There was also limited data availability on the costing information for malnutrition programs as well as the access to the nutrition programs in Nigeria. It is important to evaluate the percentage of households that have access to such programs across zones and wealth quintiles in Nigeria. This would be useful for policy implications in planning for health budget.

\section{Conclusion}

The CHE risk of malnutrition treatment in Nigeria varied across zones and wealth quintiles but highly correlated with the average consumption level for each wealth quintile in each zone. While the richest wealth quintile had almost no risk of CHE, the poorest wealth quintile, especially in northwest and northeast zones, faced higher risk of CHE, 47 to $59 \%$.

\section{Competing Interests Statement}

The authors declare that there are no competing or potential conflicts of interest.

\section{References}

Daures, M., Phelan, K., Issoufou, M., Kouanda, S., Sawadogo, O., Issaley, K., ... \& Becquet, R. (2020). New approach to simplifying and optimising acute malnutrition treatment in children aged 6-59 months: the OptiMA single-arm proof-of-concept trial in Burkina Faso. British Journal of Nutrition, 123(7), 756-767. https://doi.org/10.1017/S0007114519003258

Frankel, S., Roland, M., \& Makinen, M. (2015). Costs, cost-effectiveness, and financial sustainability of CMAM in Northern Nigeria. Field Exchange 50, 51. Retrieved from https://ciff.org/wp-content/uploads/2019/09/R4D_CMAM_CostEffectiveness_FinancialSustainability_eval uation.pdf

Mwene-Batu, P., Bisimwa, G., Baguma, M., Chabwine, J., Bapolisi, A., Chimanuka, C., ... \& Donnen, P. (2020). Long-term effects of severe acute malnutrition during childhood on adult cognitive, academic and behavioural development in African fragile countries: The Lwiro cohort study in Democratic Republic of the Congo. PLoS One, 15(12), e0244486. https://doi.org/10.1371/journal.pone.0244486

Njuguna, R. G., Berkley, J. A., \& Jemutai, J. (2020). Cost and cost-effectiveness analysis of treatment for child undernutrition in low-and middle-income countries: A systematic review. Wellcome Open Research, 5. https://doi.org/10.12688/wellcomeopenres.15781.1

Riumallo-Herl, C., Chang, A. Y., Clark, S., Constenla, D., Clark, A., Brenzel, L., \& Verguet, S. (2018). Poverty reduction and equity benefits of introducing or scaling up measles, rotavirus and pneumococcal vaccines in low-income and middle-income countries: a modelling study. BMJ global health, 3(2), e000613. https://doi.org/10.1136/bmjgh-2017-000613 
UNICEF. "Nutrition". (2021). The first 1,000 days of a child's life offer a unique window of opportunity for preventing undernutrition and its consequences. See how UNICEF targets its actions to this critical period to ensure good nutrition for every child in Nigeria. Retrieved Oct 25, 2021, from https://www.unicef.org/nigeria/nutrition

World Health Organization [WHO]. (2020). Children: improving survival and well-being. Retrieved Oct 25, 2021, from https:/www.who.int/news-room/fact-sheets/detail/children-reducing-mortality

Yerramilli, P., Fernández, Ó., \& Thomson, S. (2018). Financial protection in Europe: a systematic review of the literature and mapping of data availability. Health Policy, 122(5), 493-508. https://doi.org/10.1016/j.healthpol.2018.02.006

\section{Copyrights}

Copyright for this article is retained by the author(s), with first publication rights granted to the journal.

This is an open-access article distributed under the terms and conditions of the Creative Commons Attribution license (http://creativecommons.org/licenses/by/4.0/). 